\title{
OCORRÊNCIA DE ALTERAÇÕES OCULOPALPEBRAIS EM PACIENTES OBSERVADOS NA FACULDADE DE MEDICINA DE BOTUCATU - SÃO PAULO
}

\author{
OCULAR AND EYELID ALTERATIONS OBSERVED IN THE
}

MEDICAL SCHOOL OF BOTUCATU - SÃO PAULO, BRAZIL

Silvana A. Schellini' ; Eduardo S. Yamamoto²; Erika Hoyama ${ }^{3}$; Maria R.B. Moraes- Silva ${ }^{1}$ \& Carlos R. Padovani ${ }^{4}$

${ }^{1}$ Docente $;{ }^{2}$ Graduando; ${ }^{3}$ Residente de Oftalmologia. Departamento de Oftalmologia, Otorrinolaringologia e Cirurgia de Cabeça e Pescoço da Faculdade de Medicina de Botucatu - UNESP. Docente ${ }^{4}$. Departamento de Bioestatística - Instituto de Biociências - UNESP CorRESPONDÊNCIA: Silvana Artioli Schellini. Departamento de Oftalmologia, Otorrinolaringologia e Cirurgia de Cabeça e Pescoço, Faculdade de Medicina de Botucatu - UNESP. Cep: 18618-000 Botucatu-São Paulo-Brasil.e-mail:sartioli@fmb.unesp.br

SCHELLINI SA; YAMAMOTO ES; HOYAMA E; MORAES-SILVA MRB \& PADOVANI CR. Ocorrência de alterações oculopalpebrais em pacientes observados na Faculdade de Medicina de Botucatu-São Paulo. Medicina, Ribeirão Preto, 33: 331-337, jul./set.2000.

RESUMO: Modelo do estudo: observacional retrospectivo. Objetivo: conhecer a freqüência das alterações oculopalpebrais em pacientes atendidos na Faculdade de Medicina de Botucatu, São Paulo. Método: o estudo foi realizado através da análise de fichas de atendimento no Serviço de Plástica Ocular da Faculdade de Medicina de Botucatu, durante o período de 12 anos, avaliando-se a idade, sexo, procedência e diagnóstico principal dos pacientes atendidos. Resultados: no período estudado foram avaliados 3323 pacientes, 58,3\% dos indivíduos eram procedentes da região de Botucatu; a faixa etária superior a 60 anos $(41,6 \%)$ e o sexo feminino foram os prevalentes $(55,7 \%)$ e as patologias com alteração da posição palpebral foram as mais comuns. Comentários: as alterações mais freqüentes foram as relacionadas com a posição das pálpebras e as lesões benignas; o conhecimento da freqüência das alterações oculopalpebrais é importante para a adoção de medidas preventivas e para planejar o treinamento de novos profissionais.

UNITERMOS: Cílios. Pálpebras. Traumatismos Oculares. Neoplasias Oculares.

\section{1- INTRODUÇÃO}

As alterações oculopalpebrais são bastante freqüentes em nosso meio. Uma vez que as pálpebras e o filme lacrimal fazem parte do mecanismo protetor ocular, alterações poderão ser causa de danos para os olhos, influindo diretamente na saúde visual e podendo prejudicar o resultado de intervenções cirúrgicas intra-oculares.

A especialidade "Plástica Ocular", no presente ano, está fazendo 25 anos em nosso país e os serviços que a ela se dedicam têm se multiplicado cada vez mais, valorizando a importância das afecções e a necessidade de tratamento adequado.

Casuísticas a respeito da população atingida pelas patologias e estudos sobre a frequiência e a prevalência das alterações observadas não existem. Os relatos sobre as alterações oculoplásticas versam, na sua grande maioria, sobre etiopatogenia, técnicas operatórias e resultados obtidos. Dificilmente se encontram dados a respeito da incidência ou da prevalência das diversas afecções. Motivados por isso, resolvemos estudar a ocorrência das patologias em nosso meio, através da avaliação dos pacientes que freqüentam o nosso serviço. 


\section{2- OBJETIVO}

Conhecer a frequência das alterações oculopalpebrais em pacientes atendidos na Faculdade de Medicicna de Botucatu.

\section{3- MATERIAL E MÉTODO}

Foi realizado estudo retrospectivo dos pacientes atendidos no ambulatório de Plástica Ocular da Disciplina de Oftalmologia do Hospital das Clínicas da Faculdade de Medicina de Botucatu, no período de 1986 a 1998.

Através dessa casuística, obtivemos dados a respeito da idade, do sexo, da procedência e do diagnóstico principal dos pacientes observados.

Os pacientes foram divididos em faixas etárias: menor que 10 anos, de 10 a 19 anos, de 20 a 39 anos, de 40 a 59 anos e maior que 60 anos.

Quanto à procedência, os pacientes foram agrupados segundo região de procedência (DIR).

Em relação ao diagnóstico principal, os pacientes foram distribuídos segundo os seguintes grupos de diagnósticos: alterações na cavidade oftálmica (cavidade anoftálmica, microftalmia, phthisis bulbi), alterações nos cílios (triquíase e distiquíase), alterações palpebrais (dermatocalase, ectrópio, ptose, lagoftalmo, entrópio, blefarocalase, retração palpebral, blefaroes- pasmo essencial, epibléfaro, blefarofimose, pseudoptose, síndrome de Marcus-Gunn, coloboma, paralisia do III par), trauma (ocular, laceração palpebral e cortante da pálpebra), lesões benignas (de conjuntiva: pterígeo, nevus, cistos, dermolipoma, simbléfaro, leucoplasia, teratoma e de pálpebra: calázio, cistos, papiloma, nevus, xantelasma, hemangioma, ordéolo, ceratose, molusco) e lesões malignas (de conjuntiva: carcinoma espinocelular, melanoma, linfoma e de pálpebra: carcinoma basocelular, carcinoma meibomiano, carcinoma espinocelular, melanoma).

A partir dos dados coletados, foram obtidas as distribuições de frequiência da faixa etária, sexo, região de procedência e grupos de diagnósticos. O estudo da distribuição preferencial das variáveis abordadas foi realizado através do teste quiquadrado ${ }^{(1)}$, considerando-se o nível de $5 \%$ de significância.

\section{3- RESULTADOS}

Foram atendidos, no ambulatório de Plástica Ocular da Disciplina de Oftalmologia do Hospital das Clínicas da Faculdade de Medicina de Botucatu, 3323 pacientes, durante o período de 12 anos, sendo, a maioria significativa $(\mathrm{P}<0,0001)$, procedentes da região de Botucatu $(58,35 \%)$.

A distribuição dos pacientes, segundo grupo de patologias e faixa etária (Tabela I), mostrou que a

\begin{tabular}{|c|c|c|c|c|c|c|}
\hline \multirow{2}{*}{$\begin{array}{c}\text { GRUPOS DE } \\
\text { DIAGNÓSTICOS }\end{array}$} & \multicolumn{5}{|c|}{ FAIXA ETÁRIA } & \multirow{2}{*}{ TOTAL(\%) } \\
\hline & $<10 \mathrm{a}(\%)$ & $10-19 a(\%)$ & $20-39 a(\%)$ & $40-59 a(\%)$ & $>60 a(\%)$ & \\
\hline Cavidade & $64(1,93)$ & $37(1,11)$ & $58(1,75)$ & $60(1,81)$ & $54(1,63)$ & $273(8,23)$ \\
\hline Alterações nos cílios & $53(1,59)$ & $6(0,18)$ & $20(0,60)$ & $110(3,31)$ & $297(8,94)$ & $486(14,62)$ \\
\hline Alterações Palpebrais & $46(1,38)$ & $3(0,09)$ & $23(0,69)$ & $187(5,63)$ & $222(6,68)$ & $481(14,48)$ \\
\hline Ectrópio & $45(1,35)$ & $7(0,21)$ & $19(0,57)$ & $55(1,66)$ & $395(11,89)$ & $521(15,68)$ \\
\hline Ptose & $103(3,10)$ & $32(0,96)$ & $36(1,08)$ & $21(0,63)$ & $28(0,84)$ & $220(6,61)$ \\
\hline Entrópio & $4(0,12)$ & $2(0,06)$ & $4(0,12)$ & $27(0,81)$ & $69(2,08)$ & $106(3,20)$ \\
\hline Trauma & $18(0,54)$ & $7(0,21)$ & $15(0,45)$ & $8(0,24)$ & $2(0,06)$ & $50(1,50)$ \\
\hline Blefaroespasmo & $0(0,00)$ & $0(0,00)$ & $0(0,00)$ & $5(0,15)$ & $5(0,15)$ & $10(0,30)$ \\
\hline Lesões benignas & $159(4,78)$ & $118(3,55)$ & $245(7,37)$ & $283(8,52)$ & $235(7,07)$ & $1040(31,30)$ \\
\hline Lesões malignas & $17(0,51)$ & $0(0,00)$ & $6(0,18)$ & $38(1,14)$ & $75(2,26)$ & $136(4,09)$ \\
\hline TOTAL & $509(15,32)$ & $212(6,37)$ & $426(12,81)$ & $794(23,90)$ & $1382(41,60)$ & 3323 \\
\hline
\end{tabular}


maioria expressiva $(\mathrm{P}<0,0001)$ dos pacientes possuía idade acima de 60 anos $(41,6 \%)$ seguida da faixa entre 40 a 59 anos $(23,9 \%)$. Foi observado que o predomínio de ocorrência de tais faixas etárias deu-se, principalmente, em portadores de ectrópio, alterações nos cílios e dermatocalase. Na faixa pediátrica, a patologia mais freqüente foi a ptose palpebral congênita, seguida pelas alterações da cavidade orbitária.

Quanto à distribuição dos pacientes segundo grupos de diagnósticos: (cavidade anoftálmica, alteração dos cílios, alterações palpebrais e lesões tumorais) houve maior prevalência das alterações palpebrais (Tabela I). A avaliação da distribuição dos pacientes, segundo diagnóstico mais comum, apontou para a ocorrência de maior número de acometimento por lesões benignas (31,3\%). Em seguida, alteração dos cílios (14,6\% e, dentro do grupo das alterações palpebrais, encontrou-se o ectrópio $(15,6 \%)$ e a dermatocalasia $(14,4 \%)$.

A distribuição dos pacientes, segundo grupos de diagnósticos e sexo, mostrou, com significância $(\mathrm{P}<0,0001)$, que o sexo feminino procurou mais o serviço que o masculino $(55,76 \%$ x 44,24\%); de acordo com a patologia apresentada, as diferenças entre sexos foram mais relacionadas com alterações palpebrais (do tipo dermatocalasia) e lesões benignas da pálpebra e da conjuntiva (Tabela II).

Dos portadores de alterações na cavidade, a maioria possuía phthisis bulbi $(36,6 \%)$ e cavidade tipo $1(35,9 \%)$. Não se observou $(\mathrm{P}>0,05)$ distribuição preferencial das faixas etárias nos pacientes com alterações da cavidade (Tabela III).

As alterações dos cílios e das pálpebras (Tabela IV) predominaram significativamente $(\mathrm{P}<0,0001)$ em pacientes acima de 60 anos; das alterações nos cílios, a triquíase foi mais freqüente que a distiquíase,

\begin{tabular}{|c|c|c|c|c|c|c|}
\hline \multirow{2}{*}{$\begin{array}{c}\text { GRUPOS DE } \\
\text { DIAGNÓSTICOS }\end{array}$} & \multicolumn{5}{|c|}{ FAIXA ETÁRIA } & \multirow{2}{*}{$\begin{array}{c}\text { TOTAL } \\
(\%)\end{array}$} \\
\hline & $<10 \mathrm{a}(\%)$ & $10-19^{a}(\%)$ & $20-39 a(\%)$ & $40-59 a(\%)$ & $>60 a(\%)$ & \\
\hline C. anoftálmica 1 & 24 & 10 & 20 & 20 & 24 & 98 \\
\hline C. anoftálmica 2 & 10 & 2 & 2 & 5 & 7 & 26 \\
\hline C. anoftálmica 3 & 5 & 3 & 5 & 7 & 8 & 28 \\
\hline C. anoftálmica 4 & 1 & 1 & 1 & 2 & 2 & 7 \\
\hline Microftalmia & 9 & 2 & 3 & 0 & 0 & 14 \\
\hline Phthisis bulbi & 15 & 19 & 27 & 26 & 13 & 100 \\
\hline TOTAL & 64 & 37 & 58 & 60 & 54 & 273 \\
\hline
\end{tabular}


ocorrendo, principalmente, em indivíduos com idade acima de 60 anos, mas, também, ocorrendo em porcentagem expressiva de pacientes da faixa etária de 40 a 59 anos. Além disso, a triquíase foi mais obser- vada que a distiquíase; dermatocalasia foi o diagnóstico mais freqüente dentre as alterações palpebrais, seguida pelo ectrópio involutivo, ptose congênita e ectrópio cicatricial. Os traumas foram mais freqüen-

Tabela IV - Freqüência de casos, segundo diagnóstico de alterações em cílios, pálpebras, trauma e faixa etária

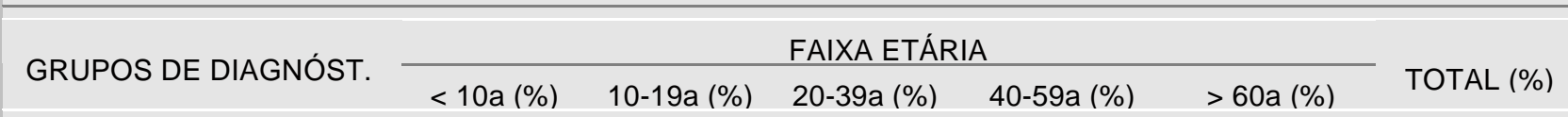

\section{Alteracões nos cílios}

Triquíase

Distiquíase

\section{Alterações Palpebrais}

Dermatocalasia

Ectrópio involutivo

Ptose congênita

Ectrópio cicatricial

Lagoftalmo

Entrópio invol.

Entrópio cicatr

Ptose involut.

Entrópio espást

Blefarocalase

Retração palp

Blefaroespasmo essencial

Epibléfaro

Ptose traumát.

Blefarofimose

Pseudoptose

S. de Marcus-Gunn

Coloboma

Paralisia III par

\section{Trauma}

Trauma ocular

T.palpebral

TOTAL

269

o

15

5

5

6

0

$$
100
$$

10

276

445

41

21
30

$$
0
$$

93

40

5

0

4

0

0

2

1

0

10

10
5

5

2

3

2

1

0

15

15

0

33

10

9

0

4

0

0

2

6

0

0

3

0

0

0

0

0
177

28

10

12

15

15

8

9

9

4

8

2

5

0

1

0

1

0

0

0

\section{1}

434

318

168

147

56

43

42

35

21

21

11

10

10

10

4

4

2

1

1

TOTAL 
tes em crianças e indivíduos de 20 a 39 anos, sendo o trauma ocular, mais freqüente que o palpebral.

A Tabela $\mathrm{V}$ mostra a distribuição das lesões benignas e malignas. Das lesões benignas, localizadas na conjuntiva, observou-se maior freqüência do pterígeo. Na pálpebra, o calázio e os cistos foram os mais frequientes. Dentre as lesões malignas, o carcinoma espinocelular foi o mais freqüente na conjuntiva

Tabela V - Freqüência de casos, segundo diagnóstico de lesões benignas e malignas e faixa etária

\begin{tabular}{|c|c|}
\hline & \\
\hline$<10 \mathrm{a}(\%)$ & $10-19 a(\%)$ \\
\hline
\end{tabular}

\section{Benignas}

\section{Conjuntiva}

Pterígeo

Nevus conj.

28

11

Cisto conj.

3

Dermolipoma

Simbléfaro

7

Leucoplasia

3

Teratoma

0

2

$\begin{array}{rr}6 & 65 \\ 11 & 5 \\ 3 & 6 \\ 4 & 2 \\ 0 & 6 \\ 0 & 1 \\ 1 & 0\end{array}$

\section{5 \\ 2 \\ 11 \\ 2 \\ 2 \\ 2}

61

235

\section{Pálpebra}

Calázio

25

Cisto

Papiloma

Nevus

13

50

50

17

13

Xantelasma

1

Hemangioma

10

Ordeólo

Ceratose

4

Molusco

2

Outros

2

0

$6 \quad 39$

56

28

39

13

13

11

4

8

2

31

11

4

2

0

\section{4}

\section{L. malignas}

\section{Conjuntiva}

Ca espinocelular

Melanoma

Linfoma

\section{Pálpebra}

Ca basocelular

Ca meibomiano

Ca espinocelular

Melanoma

45

2

1

2

0

0

1

0

0

TOTAL

$\begin{array}{ll}2 & 0 \\ 4 & 0 \\ 0 & 0\end{array}$

$\begin{array}{ll}0 & 4 \\ 0 & 0 \\ 0 & 0\end{array}$

4
0
0

37
35
43
13
4
10

37

18

186

35

50 
e o carcinoma basocelular, o mais freqüente dos tumores palpebrais. Com relação à faixa etária, a maior procura pelo serviço em decorrência de lesões benignas e malignas, fez-se por indivíduos acima de 40 anos ( $\mathrm{P}<0,0001)$, seguida de perto pelos acima de 20 anos.

\section{4- DISCUSSÃO}

A motivação deste estudo foi conhecer a frequiência das patologias que se apresentam para tratamento em serviço de Plástica Ocular.

Apesar de subespecialidade relativamente recente dentro da Oftalmologia, a Plástica Ocular tem ocupado espaço importante, principalmente pela amplitude do campo de atuação do oftalmologista que lida, em geral, com crianças e idosos, na grande maioria das vezes. Assim, as alterações involutivas, existentes inexoravelmente em todos os indivíduos, serão causas possíveis de tratamento.

A avaliação da procedência dos nossos pacientes mostra a área de abrangência do serviço, que atende parte importante do interior paulista.

Para praticidade na avaliação, classificamos as patologias em quatro grupos: cavidade anoftálmica, alterações em cílios, alterações da posição palpebral e lesões benignas e malignas. Esse tipo de classificação foi proposto por se entender que se trata de grupos de patologias específicas, com tratamento diverso entre elas.

A procura pela subespecialidade se deu, principalmente, pelas faixas etárias mais elevadas, nas quais se detectou, principalmente, o ectrópio e as alterações da posição dos cílios. O predomínio da dermatocalasia na faixa de 40 a 59 anos, ocorreu pela maior procura para correção estética nessa faixa etária, operação que visa o rejuvenescimento do aspecto dos olhos e não a modificação da sua forma básica e natural. Não foram especificadas como parte dos diagnósticos, mas as bolsas de gordura e a pseudoptose por excesso de peso, nas pálpebras superiores, freqüentemente se associam à dermatocalasia.

Na faixa pediátrica, o principal grupo de patologias foi a ptose. Pode-se considerar a ptose como um grupo, já que existem vários tipos diferentes dela.

A distribuição dos pacientes, nos grupos de patologias propostos, mostrou diferenças significativas quanto ao sexo. Assim, a procura pelo tratamento da dermatocalasia ocorreu, como o esperado, mais por mulheres. O mesmo ocorreu com as lesões palpebrais benignas. Por razões estéticas, as mulheres predominaram com relação às duas patologias. Os homens procuraram mais o serviço por lesões benignas das pálpebras, seguidas por ectrópio palpebral. Ainda com relação ao sexo, a procura por cavidade anoftálmica foi maior entre homens, em geral, mais expostos aos traumas que são causa importante de perda do bulbo ocular.

A faixa etária de portadores de cavidade anoftálmica mais afetada foi a de crianças, ressaltando-se o fato das perdas oculares na infância e as dificuldades para o tratamento da cavidade em órbitas ainda em desenvolvimento.

As alterações dos cílios ocorreram mais em idosos, sendo a causa mais comum, a inflamação crôni$\mathrm{ca}^{(2)}$. Em geral, na nossa região, decorrem do tracoma IV, que aqui é bastante prevalente.

O ectrópio se destaca dentre as alterações de posição da pálpebra, principalmente o do tipo involutivo.

Já, a casuística de traumatismo da pálpebra aqui apresentada, a nosso ver, não é real, uma vez que o tratamento ambulatorial ao trauma palpebral só é dado aos casos seqüelares e não aos portadores de trauma recente.

Das lesões conjuntivais, o pterígio foi a mais freqüente. Devido ao fato de vivermos em área subtropical, úmida, sujeita a radiações ultravioleta e fatores ambientais propícios, como poeira e vento, a lesão tem alta prevalência em nosso meio ${ }^{(3)}$.

O nevus conjuntival é lesão bastante aparente e a procura pelo tratamento é feita com frequiência, o que levou ao encontro da patologia em segundo lugar.

Na pálpebra, mais comumente, encontraram-se o calázio e os cistos.

As lesões malignas foram muito mais raras, como seria lógico esperar, sendo o carcinoma espinocelular, na conjuntiva, e o carcinoma basocelular, na pálpebra, os mais comuns, dados semelhantes aos encontrados na literatura, onde a prevalência do carcinoma basocelular varia de 70 a $90 \%{ }^{(4)}$.

As alterações observadas se referem ao atendimento de pacientes que procuram o Serviço de Plástica Ocular de um Hospital Universitário, não havendo seleção por nível socioeconômico. Contudo, os dados levantados devem sofrer uma análise crítica, uma vez que o enfoque que o Serviço tem, para atuação na Comunidade, está diretamente baseado na formação dos responsáveis pelo Serviço, fator que, sem dúvida, se reflete no atendimento a algumas patologias em detrimento de outras. 
Os dados encontrados, comparados com relato prévio $^{(5)}$, em que se estudaram 269 pacientes, residentes no estado de Pernambuco, mostram várias semelhanças no tocante à ocorrência de alterações oculopalpebrais, mesmo as duas regiões estudadas sendo tão distintas. Novos estudos devem surgir para que se possa comparar se a realidade aqui encontrada é a mesma da de outras regiões.

Além da avaliação da prevalência das patologias citadas, este estudo permite saber quais são as principais patologias para as quais os novos cirurgiões devem ser capacitados dentro da Oculoplástica.

SCHELLINI SA; YAMAMOTO ES; HOYAMA E; MORAES-SILVA MRB \& PADOVANI CR. Ocular and eyelid alterations observed in the Medical School of Botucatu - São Paulo, Brazil. Medicina, Ribeirão Preto, 33: 331-337, july./sept.2000.

ABSTRACT: Study model: observational, retrospective.Purpose :to evaluate the oculopalpebral alterations at Faculdade de Medicina de Botucatu, São Paulo State. Methods: a retrospective study in the Oculoplastic Service of the Faculdade de Medicina de Botucatu during the last 12 years was done. The age, sex, place of residence and the patients' main diagnosis were evaluated. Results: 3323 patients were assisted. $58,3 \%$ of them lived in Botucatu area. The older than 60 years $(41.6 \%)$ and the females ( $55.7 \%)$ were the prevalent group of attendance. The eyelid position alterations were the most common pathology observed. Comments: the positional eyelids disorders and the benign lesions were the manly pathologies observed The knowledge of the oculopalpebral alterations frequence is important to prevention and in order to teach new professionals who will act in this Ophthalmology area .

UNITERMS: Cilia. Eyelids. Eye Injuries. Eye Neoplasms.

\section{REFERÊNCIAS BIBLIOGRÁFICAS}

1 - STREINER DL \& NORMAN GR. Biostatistics. The base essentials. Mosby, St. Louis, 1994. 260p.

2 - ELKINGTON AR \& KHAW PT. Eyelid and lacrimal disorders. BMJ $297: 473-477,1988$.

3 - FIALHO TA. Pterígio. Rev Bras Oftalmol 47:.41-43, 1988.

4 - SCHELLINI AS ; COSTA JP; CARDILO JÁ; PARO PT; MARQUES MEA \& SILVA MRBM. Neoplasias malígnas das pálpebras na Faculdade de Medicina de Botucatu. Rev Bras Oftalmol 59:.47-53, 1990.
5 - VASCONCELOS AA \& GUERRA LHA. Perfil epidemiológico dos pacientes atendidos no Ambulatório de Cirurgia Plástica Ocular da Fundação Altino Ventura - Recife - PE. Rev Bras Oftalmol 58: 567-572, 1999.

Recebido para publicação em 26/01/2000

Aprovado para publicação em 19/07/2000 